\title{
AN AUTOMATED FRAMEWORK FOR CORONARY ANALYSIS FROM CORONARY CINE ANGIOGRAMS USING MACHINE LEARNING AND IMAGE ANALYSIS TECHNIQUES
}

\author{
K. Santhi ${ }^{1 *}$, A. Rama Mohan Reddy ${ }^{2}$ \\ ${ }^{1}$ Research Scholar, CSE Department, SV University College of Engineering \\ ${ }^{2}$ Professor, CSE Department, SV University College of Engineering \\ ${ }^{1}$ santhi.kuraganti@gmail.com, ${ }^{2}$ ramamohansvu@gmail.com
}

\begin{abstract}
Cardiovascular disease (CVD) is one of the critical diseases and the most common cause of morbidity and mortality worldwide. Therefore, early detection and prediction of such a disease is extremely essential for a healthy life. Cardiac imaging plays an important role in the diagnosis of cardiovascular disease but its role has been limited to visual assessment of heart structure and its function. However, with the advanced techniques and tools of big data and machine learning, it become easier to clinician to diagnose the CVD. Stenosis with in the Coronary Arteries (CA) are often determined by using the Coronary Cine Angiogram (CCA). It comes under the invasive image modality. CCA is the effective method to detect and predict the stenosis. In this paper a coronary analysis automation method is proposed in disease diagnosis. The proposed method includes pre-processing, segmentation, identifying vessel path and statistical analysis.
\end{abstract}

Keywords - Cardiovascular Disease, CCA, Cardiac Imaging, Machine Learning, Coronary Analysis.

\section{Introduction}

The human heart is the most important organ that pumps blood throughout the human body using the blood circulatory system, which carries oxygen and nutrients to the tissues and detaching carbon dioxide and other wastes. Cardiovascular disease (CVD) is a hazardous disease that can stop the survival. Coronary artery diseases (CAD) such as angina and myocardial dead tissue (basically known as a heart attack), stroke, heart disappointment, heart arrhythmia, hypertensive coronary illness, aortic aneurysms, valvular coronary illness, fringe supply route infection can be incorporated into CVD .

There are two categories of the disease diagnosis methods using image modalities in CA diseases. They are invasive methods and non-invasive methods. All imaging techniques which are catheter-based belong to invasive techniques and which are not catheter-based belongs to the noninvasive image modality. Angiography is an invasive technique of medical imaging. The lumen of blood vessels and organs of the body with particular interest in the heart chambers, veins, and the arteries can be visualized using angiograms [1]. These testing help to detect and identify a blockage or narrowing of the blood vessels. Patients can be treated after detecting such a situation of a blood vessel. 17.9 million people die each year from cardiovascular diseases and it is $31 \%$ of all deaths worldwide according to an estimation [2].

In developed countries, CVD becomes the most important reason for the deaths. Therefore, more accurate early detection and correct prediction of such a disease is a tremendous service for a healthy world. The most terrible conditions can be avoided using the detection and prediction of cardiovascular disease through image processing techniques with the help of expertise knowledge. It is used on minimal-invasive imaging modalities such as coronary CT angiography, cardiac magnetic resonance imaging (MRI), ultra sound, cardiac radionuclide imaging namely SPECT and PET modalities [3]. Computed Tomography Angiography (CTA), Coronary Angiogram, Digital Subtraction Angiography (DSA), Magnetic Resonance Angiography (MRA), Pulmonary Angiogram, Radionuclide Angiogram (RNA) are several types of angiograms used for diagnosing purposes. 
The remaining part of this paper is presented as follows. Section 2 mentions the reviews of the literature, Section 3 is discussion of the proposed method, Section 4 is results and finally conclusion and future work is presented in section 5 .

\section{Literature Review}

In the literature, automatic detection of blood vessels has been widely addressed by different methods. To construct a vessel probability map eigen values of the hessian matrix are used [4]. To increase the vessel detection capability of the GMF method, multi scale approach was proposed [5].

A study to determine the most suitable EC method for the automatic selection of parameters for the GMF applied to the coronary artery segmentation problem [6]. Canny edge detector into training is introduced, combining global information with local information using the CNN network [7].

4-neighborhood statistical region merging method to segment the target pixels after the multi-scale Hessian enhancement are used [8]. Gabor filters are used for segmentation in coronary arteries and are compared to the state-of-the-art approaches [9, 10], followed in efficiency by the Gaussian matched filters.

A multi scale gabor filter approach was proposed [11] for the detection of blood vessels in the retina. The blood vessels segmentation in angiographic images has been studied and summarized by many researchers [12 - 14]. Visual degradation in the cardiac images occurs due to the non uniform illumination $[15,16]$, which requires preprocessing to improve the visuality of the CCA. An ensemble model was proposed [20] for handling multiclass imbalanced data sets using machine learning.

\section{Proposed Method}

The proposed method as depicted in Fig.1. includes three subtasks namely preprocessing, segmentation, identification of vessel path and statistical coronary analysis for diagnosing the cardiovascular disease using coronary angiogram images.

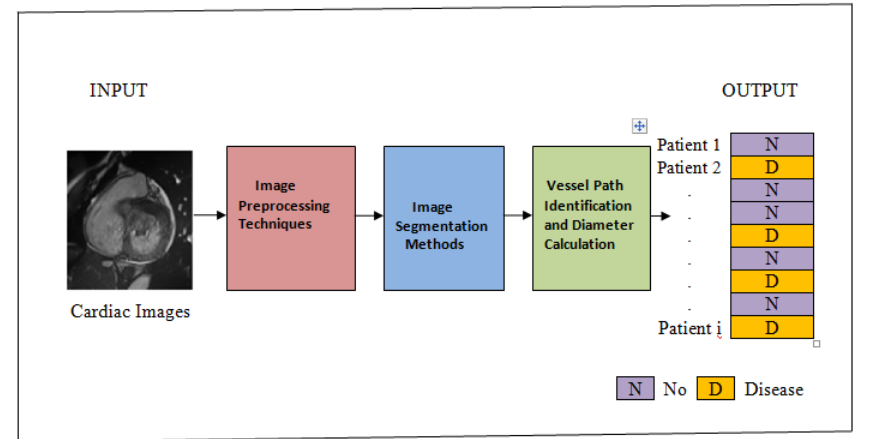

Figure 1. Automated Proposed Framework for Coronary Analysis

\section{A. Preprocessing}

The main intention of preprocessing is to improve the visual quality of CAs in the cardiac images. It can be done by reducing the visual problems from the images such as noise, low contrast, non-uniform illumination, etc. These visual degradations are shown in Fig.2. Nonuniform illumination in CCAs causes some incorrect vessel segments in computer based vessel recognition or segmentation procedures. Low contrast of the vessel may lead to a false impression of an angiographically significant lesion, which could be considered a clot [17]. Angiograms are characterized by noise, which is caused by two main sources; quantum noise and electrical noise [18][19].

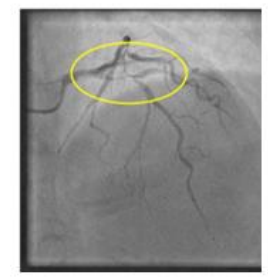

(a) Non uniform illumination

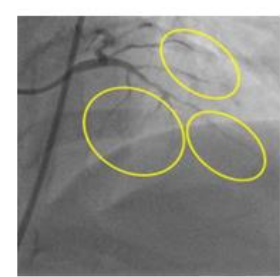

(b) Low contrast

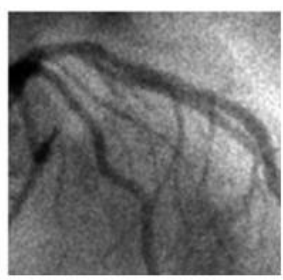

(c) Noise
Figure 2. Effect of visual degradations in CA

The frames extracted from direct CCA are given as input for preprocessing. Some of the frames are selected and will be used for visual quality enhancements which are shown in Fig.3.
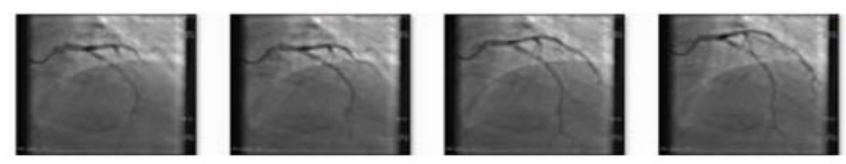

Figure 3. Selected CCA frames for Preprocessing 
The objective of preprocessing is to convert direct CCA frames into noise free uniformly illuminated frames using following procedure:

- Noise is removed from images using spatial filter and median filters.

- Homomorphic butterworth high pass filter was used to get the illumination component from the CCA frame.

1. The CCA frame is mapped into the natural logarithm domain.

2. Discrete Fourier Transform (DFT) is applied to partially processed frame to transform it into the frequency domain.

3. The butterworth high pass filter was applied to emphasize the high frequency components of the frame to be processed.

4. To filter out the high frequency bands, convolution was done between the DFT frame and the butterworth high pass filter.

5. Inverse DFT has been applied to transform the image into natural logarithm domain.

6. To get the homomorphic filtered CCA frame to the spatial domain, the transformation of natural logarithm has been inversed, which is exponential.

7. This uniformly illuminated frame has been normalized to obtain the better contrast among the vessel structures.

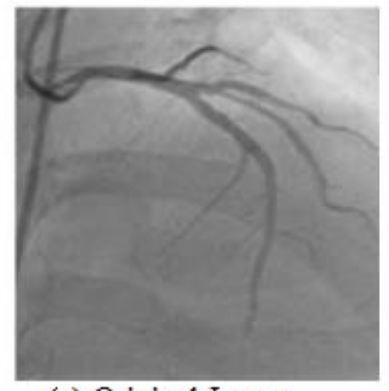

(a) Original Image

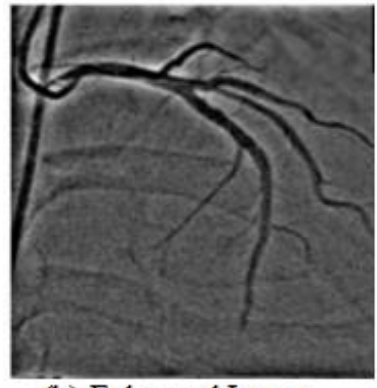

(b) Enhanced Image
Figure 4. Enhanced Image after preprocessing

\section{B. Segmentation}

After preprocessing the original image, we get a noise free and enhanced image with more uniform illumination. The enhanced image will be considered which is shown in Figure 4. and given as input for segmentation to extract coronary artery tree for identifying the vessel path and to calculate diameter for stenosis in the arteries.

The proposed coronary artery segmentation is based on multi scale gaussian matched filter(MSGMF), multi scale gabor filter(MSGF) and applying artificial neural networks(ANN) for the filters to get clear segmented vessels which is shown in Figure 5.

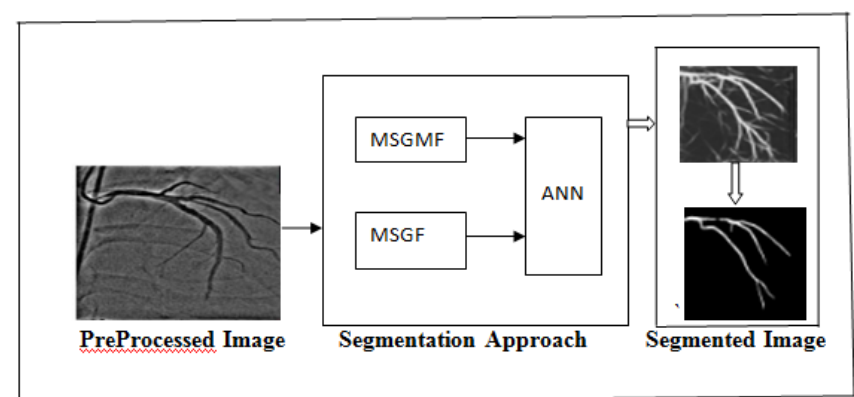

Figure 5. Proposed Segmentation Approach

MSGMF uses gaussian templates with different parameter values. Each template is intended to enhance vessels of different diameters, providing a multi scale response for the same angiogram. The single-scale Gaussian template is defined as follows:

$\operatorname{GMF}(\mathrm{x}, \mathrm{y})=-\exp \left(-\frac{\mathrm{x}^{2}}{2 \sigma^{2}}\right)\|\mathrm{y}\|<\mathrm{L} / 2$

where $\mathrm{x} \in[-\mathrm{T} / 2, \mathrm{~T} / 2]$. The parameter $\mathrm{T}$ delimits the Gaussian curve trails and produce a filter of finite width. The value of $L$ is length of a vessel segment which shares the same cross-section profile and orientation.

The MSGF, a gabor filter is a gaussian curve modulated by a sinusoid function which is capable of detecting directional features. The Gabor wavelet is to detect the edges of blood vessels in the space domain by identifying singularities in the frequency domain. Gabor filter can be defined as follows:

$\mathrm{GF}(\mathrm{x}, \mathrm{y})=\frac{1}{2 \pi \sigma x \sigma y} \exp \left[-\frac{1}{2}\left(\frac{x^{2}}{\sigma x^{2}}+\frac{y^{2}}{\sigma y^{2}}\right)\right] \cos (2 \pi f x)$

where $\sigma_{\mathrm{x}}$ and $\sigma_{\mathrm{y}}$ control the standard deviation of a two dimensional Gaussian curve in 
the $\mathrm{x}$ and $\mathrm{y}$ axis, respectively. The value of $\mathrm{f}$ determines the frequency at which the Gaussian curve is modulated. Gabor filter depends only on two parameters: $\tau$ and 1 . The diameter of the features to be detected by the Gabor filter is controlled by $\tau$, while 1 governs the elongation of a segment that shares a similar orientation.

The multi scale analysis with Gabor filters provides an improved vessel detection quality at expenses of increasing the computational time, which is mostly because of the number of image transformations between the spatial and frequency domains.

In classification problems, the prediction process of an artificial neural network might be interpreted as a sequence of projections into new feature spaces, where the linear separation of patterns represents a simpler task. In projection sequence, a weighted sum is considered for each neuron of its corresponding inputs; in addition, a bias term is usually employed to center the neuron response around a decision boundary. A multi layered perceptron network provides the coronary artery detection. The MLP performs the vessel detection by using each pixel-wise inputs from the concatenated multi scale detection response, forming the input layer of the artificial neural network. The hidden layers of the MLP take the number of neurons in each is selected by following a statistical hypothesis test for stochastic domination between a set of candidate architectures. An activation function is the sigmoid function for both hidden layers to provide a nonlinear transformation of the neuron responses. The final layer is output layer, which provides coronary artery detection response in an unbounded continuous scale by using a linear activation function.

\section{Identifying Vessel Path and Statistical Analysis}

From the segmented image, extract the entire vessel segment of interest and apply thinning procedure to identify the center line of the vessel for calculating the diameter correctly. A fast-parallel thinning algorithm widely used in image processing which has the advantages of fast speed, maintaining the connectivity of the thinned curve. Let point $\mathrm{A}$ and point $\mathrm{B}$ are intersections. Assuming that the coordinates of the intersection are $\mathrm{A}\left(\mathrm{x}_{\mathrm{a}}, \mathrm{x}_{\mathrm{b}}\right)$ and $\mathrm{B}\left(\mathrm{y}_{\mathrm{a}}, \mathrm{y}_{\mathrm{b}}\right)$, then the diameter of the blood vessel at the center point is (3).

$$
\mathrm{d}=\sqrt{\left(\left(\mathrm{x}_{\mathrm{a}}-\mathrm{x}_{\mathrm{b}}\right)^{2}+\left(\mathrm{y}_{\mathrm{a}}-\mathrm{y}_{\mathrm{b}}\right)^{2}\right)}
$$

The cardiovascular diameter measurement is mainly used to analyze the degree of stenosis of blood vessels. We have chose two blood vessels as shown in Fig.6. which are normal and diseased vessels respectively. As there is stenosis in the blood vessel-2 which corresponds to the apparent depression of the blood vessel which brings more diameter variation.

Since the digital image is discrete, the diameter is calculated using the coordinates of two pixels. However, more often, the vertical line with the centerline normal does not pass directly through the coordinate points on the two edges. Instead, the point on the edge that is close to the perpendicular is chosen. This situation can cause some errors, especially if the blood vessels are small or if the direction of the blood vessels changes. These errors cannot be avoided. As long as the measurement results can accurately reflect the trend of the blood vessel and the width of the blood vessel within the acceptable error range, it can be regarded as a proper blood vessel diameter measurement method.

We use five different performance measures to compare our segmentation results with other methods. The five performance measures are as follows: Dice Similarity Coefficient (DSC), Sensitivity (Recall), Precision, Specificity, Jaccard Similarity (JAC), and Hausdorff distance (HD). To calculate these performance measures, the true positive (TP), the true negative (TN), the false positive (FP), and the false negative (FN) need to be computed firstly. $\mathrm{TP}, \mathrm{FN}, \mathrm{FP}$, and TN denote true positive (the vessel pixels correctly identified),false negative (the vessel pixels that are not identified), false positive (the non-vessel pixels that are wrongly identified as vessel pixels), and true negative (the non-vessel pixels correctly identified) respectively.

$$
\mathrm{DSC}=\frac{2 \times T P}{(F P+T P)+(T P+F N)}
$$




$$
\begin{aligned}
& \text { Sensitivity }=\frac{T P}{T P+F N} \\
& \text { Precision }=\frac{T P}{T P+F P} \\
& \text { Specif icity }=\frac{T N}{T N+F P} \\
& \mathrm{JAC}=\frac{T P}{F P+T P+F N} \\
& \mathrm{HD}(\mathrm{X}, \mathrm{Y})=\max \left|\mathrm{d} \mathrm{XY}, \mathrm{d}_{\mathrm{Y} X}\right| \\
& \quad=\max \\
& \left\{\max _{x \in X} \min _{y \in Y} d(X, Y), \max _{y \in Y} \min _{x \in X} d(X, Y)\right\}
\end{aligned}
$$

The greater the DSC is, the higher the degree of overlap between the target vessel and the actual vessel segmented by the segmentation method. JAC is used to compare the similarity and differences between limited sample sets. If the JAC coefficient is larger, it means that their similarity is higher. Sensitivity (Recall) means correctly detected positive parts, while Specificity means correctly detected negative parts. If the Sensitivity increases, the missed segmentation rate will decrease, and if the Specificity increases, the error segmentation rate will decrease. Precision is the ratio of the number of correctly predicted parts to the total number of positive predictions, which represents how many predictions are accurate from the perspective of prediction results. Hausdorff distance is to find the similarity between two set of points. The smaller the value is, the better the segmentation result is.

\section{Results and Discussion}

The performance of our proposed method is compared with different methods based on the same images. We implemented the multi scale GMF [32] as method 1 and the multi-scale Gabor filter [33] as method 2. The segmented images of proposed method are shown in Figure 6.(b), where Figure 6.(a) represents the sample five angiogram images.

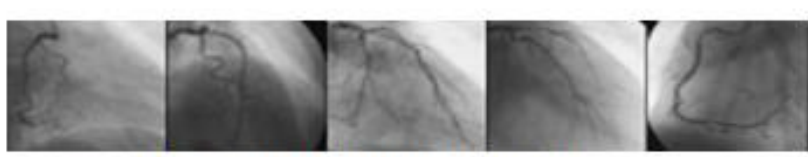

(a) Five sample angiogram images

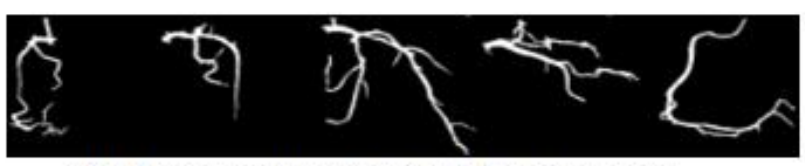

(b) Segmentation images of angiograms

\section{Figure 6. Segmentation of proposed method}

The proposed method has provided the most accurate vessel segmentation of the test set of 30 angiograms with a classification accuracy of 0.98 , and Dice coefficient of 0.93 . The results are shown in Table 1. and Figure 8. The AUC value of our proposed method for calculating diameter of vessels in diagnosing cardiovascular disease using angiograms is 0.992 which is shown in Figure 9. and Figure 10. respectively.
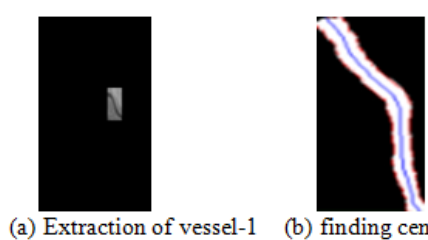

(a) Extraction of vessel-1

(b) finding center line
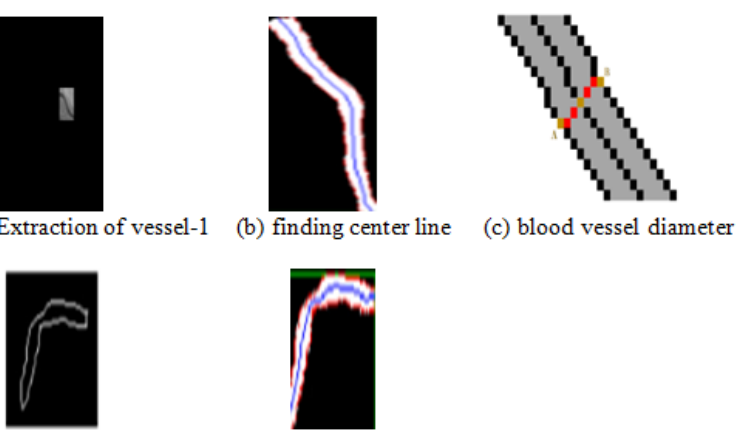

(d) Extraction of vessel-2

(e) Finding center line

Figure 7. Extraction of vessels and finding center line

Table 1. Average Recall, specificity, precision, DSC, HD,JAC of three methods of blood vessels

\begin{tabular}{|c|c|c|c|c|c|c|}
\hline Method & Recall & Specificity & Precision & DSC & HD & JAC \\
\hline Method 1 & 0.8463 & 0.9997 & 0.9254 & 0.8983 & 7.6175 & 0.8045 \\
\hline Method 2 & 0.9021 & 0.9998 & 0.9522 & 0.9246 & 4.6627 & 0.8583 \\
\hline $\begin{array}{c}\text { Proposed } \\
\text { Method }\end{array}$ & 0.9642 & 0.9996 & 0.8902 & 0.9305 & 2.5107 & 0.8721 \\
\hline
\end{tabular}




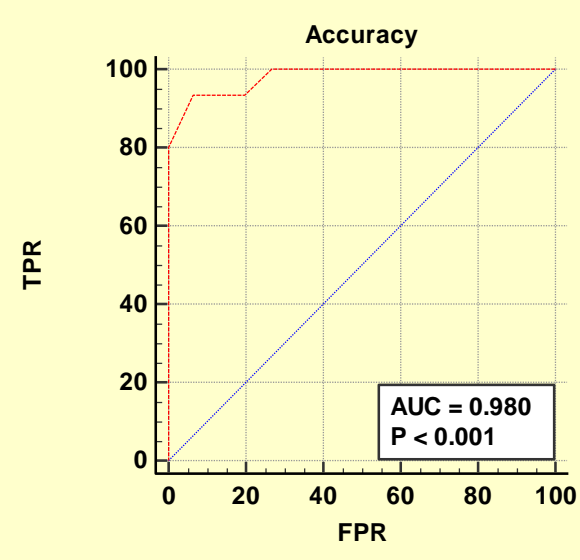

Figure 8. AUC value of proposed Segmentation Method

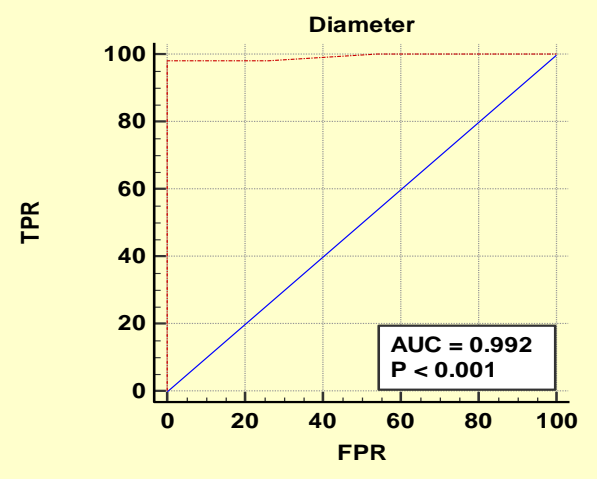

Figure 9. AUC value of Proposed Method

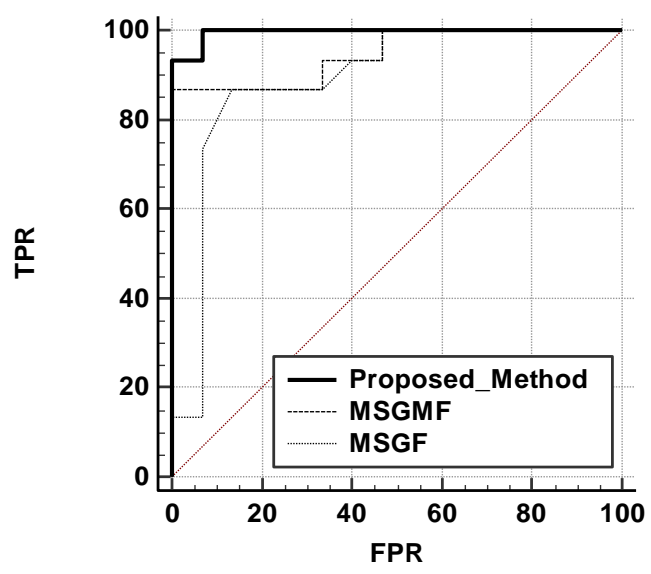

Figure 10. ROC curve of three methods

\section{Conclusion and Future Work}

This paper presents an automated framework for diagnosing cardiovascular disease in coronary arteries using coronary cine angiograms. The proposed method enhance the image through preprocessing using homomorphic butterworth high pass filter followed by segmentation task which uses multi scale Gaussian matched filter, gabor filter and multi layer perceptron for segmenting the coronary arteries.

Next a method to extract the entire vessel segment of interest and applied thinning procedure to identify the center line of the vessel for calculating the diameter was done. The results confirmed that the diameter measurement results could reflect the thickness of the blood vessel. However, because the digital image is discrete, the deviation of a pixel selection will cause the deviation of the value. So for small blood vessels, there may be variation in diameter calculation. How to calculate diameter for any type of blood vessels in better way and applying the proposed work for other image modalities can be solved in future work.

\section{References}

1. Wikipedia, "Angiography," 21 May 2019. [Online]. Available: https://en.wikipedia.org/wiki/Angiography

2. WHO, "who," [Online]. Available: http://www.who.int/cardiovascular_diseas es/en/. [Accessed 30 May 2019].

3. Z. Sun, "Cardiac Imaging Modalities in the Diagnosis of Coronary Artery Disease," in J. Clin, 2013.

4. Wang, S.; Li, B.; Zhou, S. A Segmentation Method of Coronary Angiograms Based on Multi scale Filtering and Region Growing. In Proceedings of the 2012 International Conference on Biomedical Engineering and Biotechnology, Macao, China, 28-30 May 2012; pp. 678-681.

5. Cruz-Aceves, I.; Cervantes-Sanchez, F.; Avila-Garcia, M.S. A novel multi scale Gaussian matched filter using neural networks for the segmentation of x-ray coronary angiograms.J. Healthc. Eng.2018,2018, 1-22.

6. Cruz-Aceves, I.; Hernandez-Aguirre, A.; Valdez, S.I. On the performance of nature inspired algorithms for the automatic segmentation of coronary arteries using 
Gaussian matched filters. Appl. Soft Comput.2016, 46, 665-676.

7. E. Nasr-Esfahani, N. Karimi, M. Jafari, S. Soroushmehr, S. Samavi, B.Nallamothu, and K. Najarian, "Segmentation of vessels in angiogramsusing convolutional neural networks," Biomedical Signal Processing andControl, vol. 40, pp. 240-251, 2018

8. "Automated coronary artery tree segmentation in X-ray angiography using improved Hessian based enhancement and statistical region merging," Computer Methods and Programs in Biomedicine, vol. 157, pp. 179-190, 2018

9. Cervantes-Sanchez, F.; Cruz-Aceves, I.; Hernandez-Aguirre, A.; Aviña-Cervantes, J.; Solorio-Meza, S.;Ornelas-Rodriguez, M.; Torres-Cisneros, M. Segmentation of coronary angiograms using Gabor filters and Boltzmann univariate marginal distribution algorithm. Comput. Intell. Neurosci. 2016, 2016, 1-9.

10. Cruz-Aceves, I.; Oloumi, F.; Rangayyan, R.M.; Aviña-Cervantes, J.G.; HernandezAguirre, A. Automatic segmentation of coronary arteries using Gabor filters and thresholding based on multiobjective optimization.Biomed. Signal Process. Control 2016, 25, 76-85.

11. Rangayyan, R.M.; Ayres, F.J.; Oloumi, F.; Oloumi, F.; Eshghzadeh-Zanjani, P. Detection of blood vessels in the retina with multiscale Gabor filters. J. Electron. Imaging 2008, 17, 1-7.

12. S. Moccia, E. D. Momi, S. E. Hadji, and L. S. Mattos, "Blood vessel segmentation algorithms -Review of methods, datasets and evaluation metrics," Computer Methods and Programs in Biomedicine, vol. 158, pp. 71-91, 2018.

13. C. Kirbas and F. Quek, "A review of vessel extraction techniques and algorithms," ACMComputing Surveys (CSUR), vol. 36, no. 2, pp. 81-121, 2004.

14. K. Sun, "Development of Segmentation Methods for Vascular Angiogram," IETE Technical Review, vol. 28, no. 5, p. 392, 2011.

15. S. Wang, B. Li, and S. Zhou, "A Segmentation method of coronary angiograms based on multi-scale filtering and region-growing," in lnt. Cont: on Biomedical Engineering and Biotechnology, 2012, pp. 678-681.

16. R. Xiao, 1. Yang, M. Goyal, Y. Liu, and Y. Wang, "Automatic vasculature identification in coronary angiograms by adaptive geometrical tracking," Comput. Math. Methods Med., Oct. 2013, http://dx.doi.org/1O.1155/2013/796342.

17. M. J. Kern, "Angiographic data," in Cardiac Catheterization Handbook: Expert Consult - Online and Print, 5e, 5th ed., Philadelphia, PA: Mosby, 2011, pp. 145218.

18. S. Tu , "Coronary angiography enhancement for visualization," Int J.Cardiovasc. Imaging, vol. 25, no. 7, pp. 657-667, Oct. 2009.

19. G. Sasidharan and A. George, "Frangi's vessel detection approach for coronary angiogram segmentation," Int. J. Eng. Trends Technol., vol. 13, no. 5, pp. 213217, Jul. 2014.

20. K. Santhi , A. Rama Mohan Reddy, "An Integrated Ensemble Learning Model for Multi Class Imbalanced Datasets Classification". International Journal of Advanced Science and Technology, 29(8s), 573 - 587, (2020).

\section{Authors}

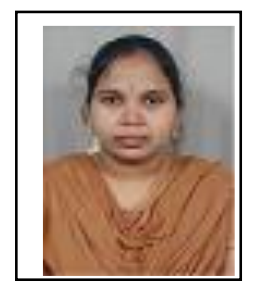

K. Santhi , received her B.Tech degree from Vignan's Engineering College, JNTUH, Guntur in 2003, M.Tech in computer Science and Engineering from JNTU, Hyderabad in 2006 and pursuing Ph.D. in Computer Science and Engineering from S V University College of Engineering, Tirupati in 2016. Her areas of interest are Big Data Analytics, Machine Learning, Data Mining.

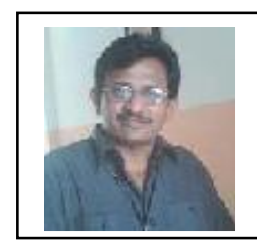

Dr. A. Rama Mohan Reddy was born in1958, received his B.Tech degree from JNT University Anantapur in 1986, Masters in Computer Science and Engineering from NIT, Warangal in 1991 and Ph.D. in Computer Science and Engineering from Sri Venkateswara University, Tirupati in 2007.He is currently working as a Professor of Computer Science and Engineering, S V University College of Engineering, Tirupati, India. His research interests are Software Engineering, Software Architecture, Cloud Computing, Operating Systems and Data Mining. 